\title{
Correction to: A class of biaxial micro/meso-scale structures for isotropic in-plane inertial sensing and actuation: design, fabrication and experiments
}

\author{
Xiaowei Shan ${ }^{1}$ (D) Jorge Angeles ${ }^{2} \cdot$ James Richard Forbes $^{2}$
}

Published online: 18 April 2020

(C) Springer-Verlag GmbH Germany, part of Springer Nature 2020

\section{Correction to: Microsystem Technologies}

https://doi.org/10.1007/s00542-020-04803-y

In the original publication, footnote mark 3, 4, 5 and their respective notes were incorrectly added to the text. The correct footnote marks and the notes are given here.

The first six mode shapes ${ }^{3}$ and frequencies are illustrated in Fig. 3. Taking Fig. 3c as an example, the suspended parts include the red proof-mass and the three light-blue connection structures between the $\Pi \Pi$ - hinges ${ }^{4}$ and the darkblue outer frame. The $\Pi \Pi$-hinges enable a periodically symmetric design with isotropic stiffness for any regular polygonal shape.

3. $t_{i}(i=x, y, z)$ denotes translation along the $i$ th-axis; $r_{i}(i=$ $z, y, x)$ denotes rotation around the out-of-plane axis and the two orthogonal in-plane axes.

4. A “ $\Pi$-hinge" is known in linkage kinematics as a $\Pi$ joint, namely, a parallelogram four-bar linkage, allowing for pure translation of one link with respect to its opposite (Wohlhart 1992; Hervé and Sparacino 1992).

The original article can be found online at https:// doi.org/10.1007/s00542-020-04803-y.

Xiaowei Shan

xiaowei.shan@mail.mcgill.ca

Jorge Angeles

angeles@cim.mcgill.ca

James Richard Forbes

james.richard.forbes@mcgill.ca

1 Beijing Key Laboratory of Performance Guarantee on Urban Rail Transit Vehicles, School of Mechanical-Electronic and Vehicle Engineering, Beijing University of Civil Engineering and Architecture, Beijing, People's Republic of China

2 McGill Centre for Intelligent Machines and Department of Mechanical Engineering, McGill University, Montreal, Canada

\subsection{Stress mitigation}

In conventional nano/microstructures, fillet design is not emphasized as it is for macro-scale structures, because etching technologies in microfabrication generate natural fillets without sharp corners or edges. In addition, the size effect of nano/microstructures makes a fillet not as smooth as expected, since the cross section is jagged with obvious material particles. Hence, an elaborate fillet design is usually not more functional than the natural fillets after microfabrication. However, in meso-scale structures, fillet design becomes important again because of the growth of the size-to-particle ratio. To improve the stress distribution and to avoid failure during operation, the Orchid W500P3 is filleted with Lamé curves, which provide $G^{2}$-continuity ${ }^{5}$ at the fillet blending points.

5. i.e., geometric continuity of point, tangent and curvature. The original article has been corrected.

Publisher's Note Springer Nature remains neutral with regard to jurisdictional claims in published maps and institutional affiliations. 